\title{
Solution of the Lane-Emden Equation Using the Bernstein Operational Matrix of Integration
}

\author{
Narayan Kumar, ${ }^{1}$ Rajesh K. Pandey, ${ }^{1}$ and Carlo Cattani² \\ ${ }^{1}$ Department of Mathematics, PDPM Indian Institute of Information Technology, Design and Manufacturing Jabalpur, Airport Road, \\ Madhya Pardish, Jabalpur 482005, India \\ ${ }^{2}$ Department of Mathematics (DIPMAT), University of Salerno, Via Ponte Don Melillo, 84084 Fisciano, Italy
}

Correspondence should be addressed to Carlo Cattani, ccattani@unisa.it

Received 28 November 2011; Accepted 22 December 2011

Academic Editors: M. Ding and J. Robertson

Copyright (C) 2011 Narayan Kumar et al. This is an open access article distributed under the Creative Commons Attribution License, which permits unrestricted use, distribution, and reproduction in any medium, provided the original work is properly cited.

Lane-Emden's equation has fundamental importance in the recent analysis of many problems in relativity and astrophysics including some models of density profiles for dark matter halos. An efficient numerical method is presented for linear and nonlinear Lane-Emden-type equations using the Bernstein polynomial operational matrix of integration. The proposed approach is different from other numerical techniques as it is based on the Bernstein polynomial integration matrix. Some illustrative examples are given to demonstrate the efficiency and validity of the proposed algorithm.

\section{Introduction}

In recent years, the studies of singular initial value problems in some special second-order ordinary differential equations (ODEs) have attracted the attention of many mathematicians and physicists. One of the most intriguing equations is the Lane-Emden-type equations which models many phenomena in mathematical physics and astrophysics. It is a nonlinear ordinary differential equation which describes the equilibrium density distribution in self-gravitating sphere of polytrophic isothermal gas and has a singularity at the origin. This equation has fundamental importance in the field of radiative cooling and modeling of clusters of galaxies. It has also proven to be most versatile in the examination of a variety of situations, including the analysis of isothermal cores, convective stellar interiors, and fully degenerate stellar configurations. Moreover, it has been recently observed [1-3] that the density profiles of dark matter halos are often modeled by the isothermal Lane-Emden equation with suitable boundary conditions at the origin. Since the solution is often given by some numerical approximation, the chosen method would implies some consequences on the physical interpretation of the dark matter evolution. In the following we will give an efficient method for computing its numerical solution.

Lane-Emden's equations $[4,5]$ are categorized as nonlinear ordinary differential equations with singular initial values. The more general Cauchy problem in this category is the following equation:

$$
y^{\prime \prime}(t)+\frac{\alpha}{t} y^{\prime}(t)+f(t, y)=g(t), \quad \alpha, t \geq 0,
$$

with initial conditions (ICs)

$$
y(0)=a, \quad y^{\prime}(0)=0,
$$

where primes denote differentiation with respect to $t, \alpha$ is constant, and $f(t, y)$ is a nonlinear function of $t$ and $y$.

It has been shown [6] that there exists an analytic solution of (1), (2) in the neighbourhood of the singular point $t=0$.

In the special case, where $\alpha=2, f(t, y)=f(y), g(t)=$ 0 and IC (2) holds, we have one of the most studied cases

$$
y^{\prime \prime}(t)+\frac{2}{t} y^{\prime}(t)+f(y)=0, \quad t \geq 0
$$


For instance, with $f(y)=y^{n}$ and $a=1$, we get

$$
y^{\prime \prime}(t)+\frac{2}{t} y^{\prime}(t)+y^{n}=0, \quad t \geq 0,
$$

which in the form

$$
\frac{1}{t^{2}} \frac{d}{d t}\left(t^{2} \frac{d y}{d t}\right)+y^{n}=0
$$

subject to IC

$$
y(0)=1, \quad y^{\prime}(0)=0
$$

was originally given by Lane [4] and (later) Emden [5].

The parameter $n$ has physical significance only in the range $0 \leq n \leq 5$. The solution for a given index $n$ is known as polytropic index $n$. Equation (3) with IC (5) has well-known analytical solutions [7] for $n=0,1,5$ while, for other values of $n$, numerical solutions are still sought. The series solution can be found by perturbation techniques and Adomian decomposition methods (ADM). However, these solutions are often convergent in restricted regions. Thus, some techniques such as the Padé method are required to enlarge the convergent regions $[8,9]$.

Similarly, by choosing $f(t, y)=e^{y}$ and $a=0$ in $\left(1^{\prime}\right)$ and (2), isothermal gas spheres equation are modeled by

$$
y^{\prime \prime}(t)+\frac{2}{t} y^{\prime}(t)+e^{y(t)}=0, \quad t \geq 0
$$

with IC

$$
y(0)=0, \quad y^{\prime}(0)=0
$$

A number of methods have recently been proposed to solve $\left(1^{\prime}\right),(6)$. They are quasilinearization methods [10-12], a piecewise linearization technique [13], and the Lagrangianbased analytic solution [14]. The approximate solutions were also given by homotopy analysis method (HAM) [15, 16], variational iteration method [17], and variational approach method [18].

A numerical method based on conversion into integral equations solved by Legendre wavelets is given in [19]. Hybrid functions have also been used in [20] to find the numerical solutions of (1) for some particular nonlinear cases.

In [21] the transform $t=e^{x}$ to $\left(1^{\prime}\right)$ is given to get

$$
\ddot{y}(x)+\dot{y}(x)+e^{2 x} f(y(x))=0,
$$

subject to the conditions

$$
\operatorname{Lim}_{x \rightarrow-\infty} y(x)=a, \quad \lim _{x \rightarrow-\infty} e^{-x} \dot{y}(x)=0,
$$

where dots denote differentiations with respect to $x$. Then, an approximate solution of (8) is obtained in $[0,1]$ by variational iteration method, for special cases when $f(y)=$ $y^{n}$ and $n=0,1,5$.

Legendre's spectral method for solving only singular IVPs is given in [22]. In [23], modified homotopy analysis methods (MHAMs) enable to obtain approximate solution and to show that MHAM solution contains the previous solutions obtained by ADM and HPM.

A collocation method based on Chebyshev's polynomials is proposed in [24]. In [25-27], three different methods are presented, to solve (1), based on the Hermite function collocation method, the Lagrangian method, and radial basis function approximation, respectively. The Jacobi-Gauss collocation method is given in [28]. In [29] the optimal homotopy asymptotic method is applied to obtain the analytic solution of singular Lane-Emden-type equation. The perturbation technique and delta-expansion method are presented in [30,31], respectively.

The aim of the present paper is to apply the Bernstein polynomial operational matrix of integration for the first time, to propose a reliable numerical technique for solving linear and nonlinear Lane-Emden's equations. Some special cases of the problem are solved to show its validity and efficiency in comparison with other existing numerical methods. The approximate solution, obtained by the proposed method, shows its superiority on the other existing numerical solution.

\section{Bernstein's Polynomials}

A Bernstein polynomial [32] is a polynomial in the Bernstein form that is a linear combination of the Bernstein basis polynomials. The Bernstein basis polynomials of degree $n$ are defined by

$$
B_{i, n}(t)=\left(\begin{array}{c}
n \\
i
\end{array}\right) t^{i}(1-t)^{n-i}, \quad \text { for } i=0,1,2, \ldots, n .
$$

There are $(n+1) n$th degree Bernstein basis polynomials forming a basis for the linear space $V_{n}$ consisting of all polynomials of degree less than or equal to $n$ in $\mathbf{R}[t]-$ the ring of polynomials over the field $\mathbf{R}$. For mathematical convenience, we usually set $B_{i, n}=0$ if $i<0$ or $i>n$. Any polynomial $B(t)$ in $V_{n}$ may be written as

$$
B(t)=\sum_{i=0}^{n} \beta_{i} B_{i, n}(t)
$$

Then $B(t)$ is called a polynomial in the Bernstein form or the Bernstein polynomial of degree $n$. The coefficients $\beta_{i}$ are called Bernstein's or Bezier's coefficients. Often, the Bernstein basis polynomials $B_{i, n}(t)$ are called the Bernstein polynomials. We will follow this convention as well. A function $f \in L^{2}[0,1]$ may be written as

$$
f(t)=\lim _{n \rightarrow \infty} \sum_{i=0}^{n} c_{\text {in }} B_{\text {in }}(t),
$$

where $c_{\text {in }}=\left\langle c, B_{\text {in }}\right\rangle$ and $\langle$,$\rangle is the standard inner product$ on $L^{2}[0,1]$.

If (3) is truncated at $n=m^{\prime}$, then we have

$$
f \cong \sum_{i=0}^{m} c_{\mathrm{im}} B_{\mathrm{im}}=C^{T} \Psi(t)
$$


where $C$ and $\Psi(t)$ are $\left(m^{\prime}+1\right) \times 1$ matrices given by

$$
\begin{gathered}
C=\left[\begin{array}{llll}
c_{0, m^{\prime}} & c_{1, m^{\prime}} & \cdots & c_{m^{\prime}, m^{\prime}}
\end{array}\right]^{T}, \\
\Psi(t)=\left[\begin{array}{llll}
B_{0, m^{\prime}}(t) & B_{1, m^{\prime}}(t) & \cdots & B_{m^{\prime}, m^{\prime}}(t)
\end{array}\right]^{T} .
\end{gathered}
$$

For taking the collocation points, let $t_{0}$ be any point near to zero and other point as follows:

$$
t_{i}=t_{0}+\frac{i}{m^{\prime}+1}, \quad i>0
$$

Let us use the notation $m=m^{\prime}+1$, for defining the Bernstein operational matrix $\Phi_{m \times m}$ as follows:

$$
\Phi_{m \times m}=\left[\begin{array}{llll}
B_{0, m^{\prime}}\left(t_{0}\right) & B_{1, m^{\prime}}\left(t_{1}\right) & \cdots & B_{m^{\prime}, m^{\prime}}\left(t_{m^{\prime}}\right)
\end{array}\right]^{T} .
$$

For example, when $m=6$, the Bernstein operational matrix is expressed as

$$
\begin{aligned}
& \Phi_{m \times m} \\
& =\left[\begin{array}{llllll}
0.0060 & 0.4019 & 0.2622 & 0.0930 & 0.0162 & 0.0006 \\
0.0000 & 0.2024 & 0.3292 & 0.2334 & 0.0816 & 0.0079 \\
0.0000 & 0.0544 & 0.2205 & 0.3125 & 0.2185 & 0.0528 \\
0.0000 & 0.0082 & 0.0830 & 0.2353 & 0.3292 & 0.1995 \\
0.0000 & 0.0007 & 0.0167 & 0.0945 & 0.2646 & 0.4019 \\
0.0000 & 0.0000 & 0.0014 & 0.0158 & 0.0886 & 0.3373
\end{array}\right] .
\end{aligned}
$$

\section{Block Pulse Function and Operational Matrix of Integration}

a set of block pulse functions (BPFs) is defined on $[0,1)$ as

$$
b_{i}(t)=\left\{\begin{array}{ll}
1, & \frac{i}{m} \leq t<\frac{i+1}{m}, \\
0, & \text { otherwise, }
\end{array} \quad \text { where } i=0,1, \ldots, m-1 .\right.
$$

The functions $b_{i}(t)$ are disjoint and orthogonal, that is,

$$
\begin{gathered}
b_{i}(t) b_{j}(t)= \begin{cases}0, & i \neq j, \\
1, & i=j,\end{cases} \\
\int_{0}^{1} b_{i}(t) b_{j}(t) d t= \begin{cases}0, & i \neq j, \\
\frac{1}{m}, & i=j .\end{cases}
\end{gathered}
$$

The block pulse operational matrix of the integration $F^{\alpha}$ is defined [33] as following:

$$
\left(I^{\alpha} B_{m}\right)(t) \approx F^{\alpha} B_{m}(t)
$$

where $I^{\alpha}=\overbrace{\iiint \cdots}^{\alpha \text { times }}$

$$
\begin{gathered}
B_{m}(t)=\left[\begin{array}{lllll}
b_{0}(t) & b_{1}(t) & \cdots & b_{m-1}(t)
\end{array}\right], \\
F^{\alpha}=\frac{1}{m^{\alpha}} \frac{1}{\Gamma(\alpha+2)}\left[\begin{array}{ccccc}
1 & \varepsilon_{1} & \varepsilon_{2} & \cdots & \varepsilon_{m-1} \\
0 & 1 & \varepsilon_{1} & \cdots & \varepsilon_{m-2} \\
0 & 0 & 1 & \cdots & \varepsilon_{m-3} \\
0 & 0 & 0 & \ddots & \vdots \\
0 & 0 & 0 & 0 & 1
\end{array}\right],
\end{gathered}
$$

with

$$
\varepsilon_{k}=(k+1)^{\alpha+1} 2 k^{\alpha+1}+(k-1)^{\alpha+1} .
$$

In general the operational matrix of integration of the vector $\psi_{m}(t)$ can be obtained as

$$
\int_{0}^{t} \psi_{m}(\tau) d \tau \approx P_{m \times m} \psi_{m}(t)
$$

where $P$ is the $m \times m$ operational matrix for integration.

The Bernstein polynomial can also be expanded and approximated into an $m$-term block pulse function (BPF) as

$$
\psi_{m}(t)=\Phi_{m \times m} B_{m}(t)
$$

Let us consider that the matrix $P_{m \times m}^{\alpha}$ is the Bernstein polynomial operational matrix of the integration, then we have

$$
\left(I^{\alpha} \psi_{m}\right)(t) \approx P_{m \times m}^{\alpha} \psi_{m}(t)
$$

Now, we have

$$
\begin{aligned}
& \left(I^{\alpha} \psi_{m}\right)(t) \approx\left(I^{\alpha} \Phi_{m \times m} B_{m}\right)(t) \\
& \quad=\Phi_{m \times m}\left(I^{\alpha} B_{m}\right)(t) \approx \Phi_{m \times m} F^{\alpha} B_{m}(t) .
\end{aligned}
$$

From (25) and (18) we get

$$
P_{m \times m}^{\alpha} \psi_{m}(t) \approx \Phi_{m \times m} F^{\alpha} \Phi_{m \times m}^{-1} \psi_{m}(t) .
$$

Then, the Bernstein polynomial operational matrix of the integration $P_{m \times m}^{\alpha}$ is given by

$$
P_{m \times m}^{\alpha}=\Phi_{m \times m} F^{\alpha} \Phi_{m \times m}^{-1}
$$

\section{Outline of the Method}

In this section, the method presented in Section 3 is applied to solve the linear and nonlinear Lane-Emden equations. Letting

$$
F(t, y)=f(y)=y^{n}(t) \quad \text { with } a=1,
$$


TABLE 1: Comparison of the numerical solution and error obtained by present method for $n=3$ in (36) with series solution [25].

\begin{tabular}{ccccc}
\hline$x$ & & Our method & $m=256$ & Series method [25] \\
\hline 0.0 & $0.00 E+00$ & $m=128$ & $0.00 E+00$ & $0.00 E+00$ \\
0.1 & $4.02 E-05$ & $0.00 E+00$ & $6.28 E-07$ & $1.40 E-06$ \\
0.5 & $2.89 E-05$ & $1.51 E-06$ & $4.96 E-07$ & $2.99 E-06$ \\
1.0 & $4.39 E-05$ & $3.79 E-05$ & $3.76 E-05$ & $1.99 E-06$ \\
\hline
\end{tabular}

$\left(1^{\prime}\right)$ can be written as

$y^{\prime \prime}(t)+\frac{2}{t} y^{\prime}(t)+y^{n}(t)=0, \quad$ with IC $y(0)=1, y^{\prime}(0)=0$

where $t, n \geq 0$.

Since exact solutions for the case $n=0,1,5$ are known, we solve them first by the proposed algorithm developed in Section 3.

Let

$$
D^{2} y(t)=k_{m}^{T} \psi_{m}(t)
$$

where $k_{m}^{T}$ is unknown,

$$
\begin{aligned}
& \Longrightarrow D y(t)=k_{m}^{T} P_{m \times m}^{1} \psi_{m}(t), \\
& \Longrightarrow y(t)=k_{m}^{T} P_{m \times m}^{2} \psi_{m}(t) .
\end{aligned}
$$

We have

$$
y(t)=k_{m}^{T} P_{m \times m}^{2} \Phi_{m \times m} B_{m}(t)+1 .
$$

Assume

$$
\begin{gathered}
k_{m}^{T} P_{m \times m}^{2} \Phi_{m \times m}+1=\left[\begin{array}{llll}
a_{1} & a_{2} & \cdots & a_{m}
\end{array}\right], \\
{[y(t)]^{n}=\left[\begin{array}{llll}
a_{1}^{n} & a_{2}^{n} & \cdots & a_{m}^{n}
\end{array}\right] B_{m}(t) .}
\end{gathered}
$$

Substituting (31)-(34) in (30), we get

$$
\begin{gathered}
k_{m}^{T} \Phi_{m \times m} B_{m}(t)+\frac{2}{t} k_{m}^{T} P_{m \times m}^{1} \Phi_{m \times m} B_{m}(t) \\
+\left[\begin{array}{llll}
a_{1}^{n} & a_{2}^{n} & \cdots & a_{m}^{n}
\end{array}\right] B_{m}(t)=0 .
\end{gathered}
$$

Solution of (35) yields the value of $k_{m}^{T}$.

\section{Numerical Results and Discussions}

In this section some special cases of $\left(1^{\prime}\right)$ have been considered to illustrate the efficiency of the method.

5.1. Standard Lane-Emden Equation. Consider the standard Lane-Emden equation:

$$
y^{\prime \prime}(t)+\frac{2}{t} y^{\prime}(t)+y^{n}(t)=0
$$

with initial condition $y(0)=1$ and $y^{\prime}(0)=0$, which has the exact solution for the case $n=0,1$, and 5 are

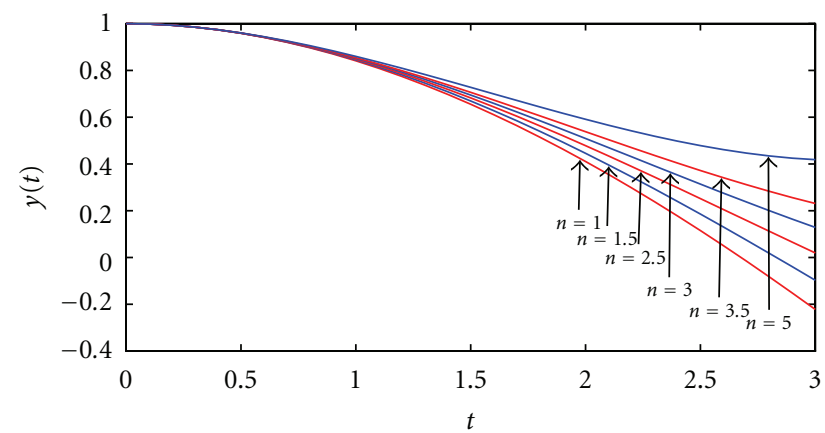

Figure 1: Graph of standard Lane-Emden's equation and its approximate solution at different values of $n=1,1.5,2.5,3,3.5,5$ (at $m=32$ ) for Section 5.1.

known. Equation (36) with the initial conditions has been solved with the proposed method, and obtained results are presented in Figure 1 along with Table 1.

Here we can see that the function basically follows the same form as that for an index $n=0$, with a few minor differences; however, the polytrope of index $n=0$ also terminates at a finite radius just as is observed in the relation for a polytrope of index $n=1$. Though these two solutions for $n=1$ and $n=0$ share many characteristics, the solution for the polytrope of index $n=5$ contains some radically different and unexpected characteristics. In this case the behavior of the function is markedly different than that of its predecessors. Here the density of the star initially decreases rapidly as radius increases but slows rapidly once a $t$-value of around three is reached. At this point the decrease slows continually. Though it may not be apparent on the graphic provided, the function never reaches 0 . It is, therefore, evident that a polytropic star of index $n=5$ has an infinite radius, and in reality cannot exist.

5.2. Isothermal Gas Spheres Equation. Letting $f(t, y)=e^{y}$ with $a=0$, (1) can be written as

$$
y^{\prime \prime}(t)+\frac{2}{t} y^{\prime}(t)+e^{y}=0
$$

with IC $y(0)=0, y^{\prime}(0)=0$. The isothermal gas spheres are modeled in [6]. Equation (38) is solved with the presented method, and the obtained solution is compared with the existing solutions. The plot of proposed and exact solution is given in Figure 2 and comparison has been done in Table 2. 
TABLE 2: Comparison of the numerical solution and error obtained by present method with series solution [25].

\begin{tabular}{ccccc}
\hline$x$ & & Our method & Series method [25] \\
\hline 0.0 & $m=32$ & $m=128$ & $m=256$ & $0.00 E+00$ \\
0.1 & $4.05 E+00$ & $0.00 E+00$ & $0.00 E+00$ & $5.85 E-07$ \\
0.2 & $4.00 E-05$ & $2.53 E-06$ & $6.32 E-07$ & $6.04 E-07$ \\
0.5 & $3.65 E-05$ & $2.50 E-06$ & $6.26 E-07$ & $5.58 E-07$ \\
1.0 & $2.51 E-05$ & $2.28 E-06$ & $5.72 E-07$ & $8.20 E-07$ \\
\hline
\end{tabular}

TABLE 3: Comparison of the numerical solution and error obtained by present method with series solution [25].

\begin{tabular}{ccccc}
\hline$x$ & & Present method & Series method [25] \\
\hline 0.0 & $0.00 E+00$ & $m=128$ & $0.00 E+00$ & $0.00 E+00$ \\
0.1 & $4.66 E-04$ & $0.00 E+00$ & $7.28 E-06$ & $3.94 E-06$ \\
0.5 & $1.46 E-04$ & $2.91 E-05$ & $2.29 E-07$ & $3.02 E-06$ \\
1.0 & $8.60 E-05$ & $9.16 E-06$ & $1.28 E-08$ & $9.31 E-07$ \\
\hline
\end{tabular}

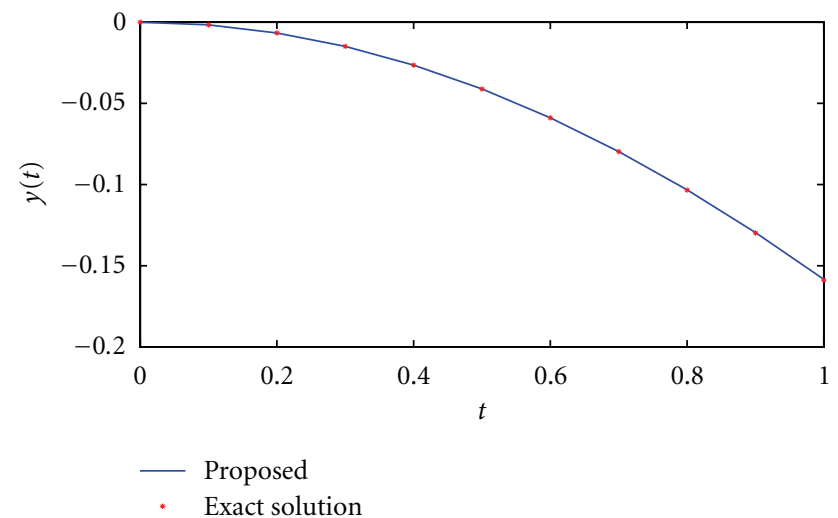

FIGURE 2: Graph of approximate solution in comparison with [9] solution for Section 5.2.

5.3. Nonlinear Homogeneous Lane-Emden Equation. Let $f(t, y)=4\left(2 e^{y}+e^{y / 2}\right)$ with $a=0$, (1) be written as

$$
y^{\prime \prime}(t)+\frac{2}{t} y^{\prime}(t)+4\left(2 e^{y}+e^{y / 2}\right)=0, \quad t \geq 0
$$

with IC $y(0)=0$ and $y^{\prime}(0)=0$, where the exact solution is $y(t)=-\operatorname{In}\left(1+t^{2}\right)$. We solve the above problem, by applying the technique described in Section 4 with $m=32,128$ and 256 and plotted in Figures 3 and 4, and comparison has been done in Table 3.

\section{Conclusions}

The Bernstein polynomial operational matrix of integrations has been applied for solving one of the most popular and intriguing differential equations, that is, the Lane-Emden equations. These results are useful in a few respects and deal with some actual state equation for stars. Though these two solutions for $n=1$ and $n=0$ share many characteristics, the solution for the polytrope of index $n=5$ contains some

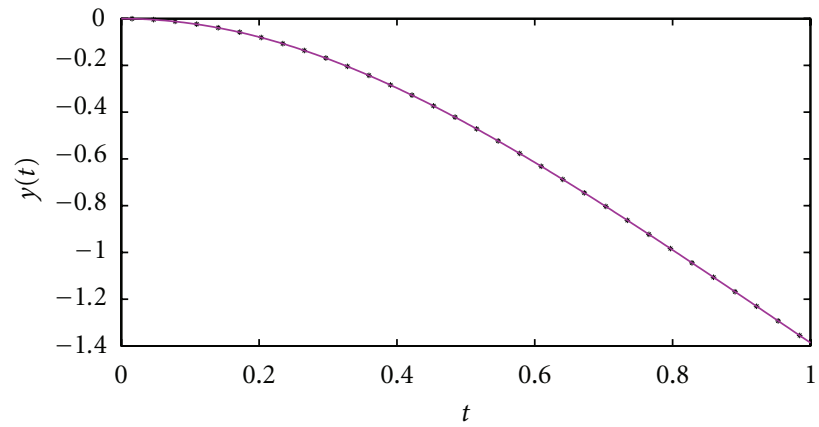

* Exact solution

Proposed

FIGURE 3: Graph of exact solution and approximate solution (at $m=32$ ) for Section 5.3.

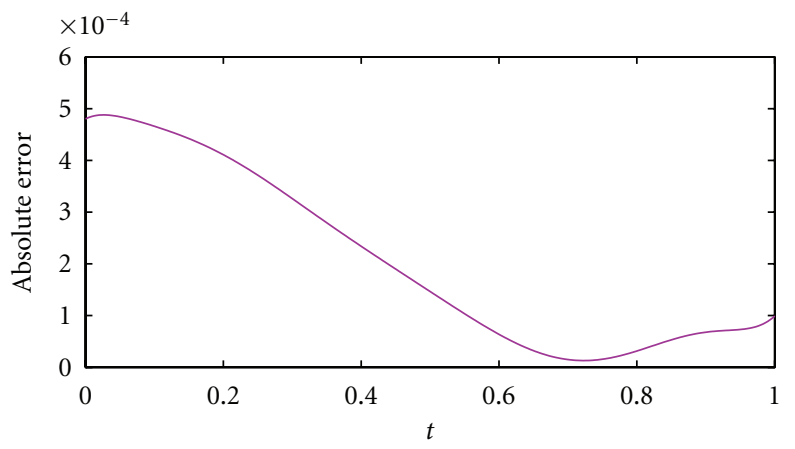

Figure 4: Absolute error for $m=32$.

radically different and unexpected characteristics. In this case the behavior of the function is markedly different than that of its predecessors. Here the density of the star $\rho=\lambda y^{n}$, where $\lambda$ represents the central density of the star and $y$ that of a related dimensionless quantity, initially decreases rapidly as radius increases but slows rapidly once a $t$-value of around 
three is reached. At this point the decrease slows continually. Though it may not be apparent on the graphic provided, the function never reaches 0 . It is, therefore, evident that a polytropic star of index $n=5$ has an infinite radius and in reality cannot exist. Despite this fact, such a model provides important theoretical perspective concerning the theory, as one may view this as the border between polytropic one that are physically feasible. It is also of interest to note that such a stellar model has, in spite of the infinite radius, a finite mass. Additionally, other stellar models, which are created in a "layered" fashion where each layer consists of a polytrope of a different index, may also utilize this function for a portion of the star, in which case a finite radius would be possible. In addition to these relations, there are also a number of other conclusions that one can draw from the polytropic model of stars. For relations of this type, there exists a relation between the polytropic index, mass of a star, and the radius. It is perhaps evident in the discussion of the analytic solutions of the polytropic index that one could possibly infer a relation between the polytropic index of the star and the radius that one would calculate from that star. In the attempt to find a relation, the most immediate result is obtained from the simple equations of stellar state.

\section{References}

[1] N. Riazi and M. R. Bordbar, "Generalized Lane-Emden equation and the structure of galactic dark matter," International Journal of Theoretical Physics, vol. 45, no. 3, pp. 495-510, 2006.

[2] H. J. de Vega and N. G. Sanchez, "Model-independent analysis of dark matter points to a particle mass at the $\mathrm{keV}$ scale," Monthly Notices of the Royal Astronomical Society, vol. 404, no. 2, pp. 885-894, 2010.

[3] G. E. Marsh, "Dark matter and charged exotic dust," In press, http://arxiv.org/abs/1107.0315.

[4] J. H. Lane, "On theoretical temperature of the sun under the hypothesis of a gaseous mass maintaining its internal heat and depending on the laws of gases known to terrestrial experiment," The American Journal of Science and Arts, vol. 50, pp. 57-74, 1870.

[5] R. Emden, Gaskugeln: Anwendungen der Mechanischen Wärmetheorie auf Kosmologische und Meteorologische Probleme, Teubner, Berlin, Germany, 1907.

[6] H. T. Davis, Introduction to Nonlinear Differential and Integral Equations, Dover, New York, NY, USA, 1962.

[7] S. Chandrasekhar, Introduction to Study of Stellar Structure, Dover, New York, NY, USA, 1967.

[8] N. T. Shawagfeh, "Nonperturbative approximate solution for Lane-Emden equation," Journal of Mathematical Physics, vol. 34, no. 9, pp. 4364-4369, 1993.

[9] A. M. Wazwaz, "A new algorithm for solving differential equations of Lane-Emden type," Applied Mathematics and Computation, vol. 118, no. 2-3, pp. 287-310, 2001.

[10] V. B. Mandelzweig and F. Tabakin, "Quasilinearization approach to nonlinear problems in physics with application to nonlinear ODEs," Computer Physics Communications, vol. 141, no. 2, pp. 268-281, 2001.

[11] R. Krivec and V. B. Mandelzweig, "Numerical investigation of quasilinearization method in quantum mechanics," Computer Physics Communications, vol. 138, no. 1, pp. 69-79, 2001.
[12] R. Krivec and V. B. Mandelzweig, "Quasilinearization approach to computations with singular potentials," Computer Physics Communications, vol. 179, no. 12, pp. 865-867, 2008.

[13] J. I. Ramos, "Linearization methods in classical and quantum mechanics," Computer Physics Communications, vol. 153, no. 2, pp. 199-208, 2003.

[14] C. M. Khalique and P. Ntsime, "Exact solutions of the LaneEmden-type equation," New Astronomy, vol. 13, no. 7, pp. 476-480, 2008.

[15] S. J. Liao, "A new analytic algorithm of Lane-Emden type equations," Applied Mathematics and Computation, vol. 142, no. 1, pp. 1-16, 2003.

[16] R. A. Van Gorder and K. Vajravelu, "Analytic and numerical solutions to the Lane-Emden equation," Physics Letters, Section A, vol. 372, no. 39, pp. 6060-6065, 2008.

[17] A. Yildirim and T. Öziş, "Solutions of singular IVPs of LaneEmden type by the variational iteration method," Nonlinear Analysis, Theory, Methods and Applications, vol. 70, no. 6, pp. 2480-2484, 2009.

[18] J. H. He, "Variational approach to the Lane-Emden equation," Applied Mathematics and Computation, vol. 143, no. 2-3, pp. 539-541, 2003.

[19] S. A. Yousefi, "Legendre wavelets method for solving differential equations of Lane-Emden type," Applied Mathematics and Computation, vol. 181, no. 2, pp. 1417-1422, 2006.

[20] H. R. Marzban, H. R. Tabrizidooz, and M. Razzaghi, "Hybrid functions for nonlinear initial-value problems with applications to Lane-Emden type equations," Physics Letters, Section A, vol. 372, no. 37, pp. 5883-5886, 2008.

[21] M. Dehghan and F. Shakeri, "Approximate solution of a differential equation arising in astrophysics using the variational iteration method," New Astronomy, vol. 13, no. 1, pp. 53-59, 2008.

[22] H. Adibi and A. M. Rismani, "On using a modified Legendrespectral method for solving singular IVPs of Lane-Emden type," Computers and Mathematics with Applications, vol. 60, no. 7, pp. 2126-2130, 2010.

[23] O. P. Singh, R. K. Pandey, and V. K. Singh, "An analytic algorithm of Lane-Emden type equations arising in astrophysics using modified Homotopy analysis method," Computer Physics Communications, vol. 180, no. 7, pp. 11161124, 2009.

[24] C. Yang and J. Hou, "A Numerical Method for Lane-Emden Equations Using Chebyshev Polynomials and the Collocation Method," in Proceedings of the IEEE International Conference on Computational and Information Sciences, pp. 97-100, 2010.

[25] K. Parand, M. Dehghan, A. R. Rezaei, and S. M. Ghaderi, "An approximation algorithm for the solution of the nonlinear Lane-Emden type equations arising in astrophysics using Hermite functions collocation method," Computer Physics Communications, vol. 181, no. 6, pp. 1096-1108, 2010.

[26] K. Parand, A. R. Rezaei, and A. Taghavi, "Lagrangian method for solving LaneEmden type equation arising in astrophysics on semi-infinite domains," Acta Astronautica, vol. 67, no. 7-8, pp. 673-680, 2010.

[27] K. Parand, S. Abbasbandy, S. Kazem, and A. R. Rezaei, "An improved numerical method for a class of astrophysics problems based on radial basis functions," Physica Scripta, vol. 83, no. 1 , article $015011,2011$.

[28] A. H. Bhrawy and A. S. Alofi, "A Jacobi-Gauss collocation method for solving nonlinear Lane-Emden type equations," Communications in Nonlinear Science and Numerical Simulation, vol. 17, no. 1, pp. 62-70, 2012. 
[29] S. Iqbal and A. Javed, "Application of optimal homotopy asymptotic method for the analytic solution of singular LaneEmden type equation," Applied Mathematics and Computation, vol. 217, no. 19, pp. 7753-7761, 2011.

[30] R. A. Van Gorder, "Exact first integrals for a Lane-Emden equation of the second kind modeling a thermal explosion in a rectangular slab," Celestial Mechanics and Dynamical Astronomy, vol. 109, pp. 137-145, 2011.

[31] R. A. Van Gorder, "An elegant perturbation solution for the Lane-Emden equation of the second kind," New Astronomy, vol. 16, no. 2, pp. 65-67, 2011.

[32] S. Bernstein, "Démonstration du théorème de Weierstrass fondée sur le calcul des probabilities," Communications of the Kharkov Mathematical Society, vol. 13, pp. 1-2, 1912.

[33] A. Kilicman and Z. A. A. Al Zhour, "Kronecker operational matrices for fractional calculus and some applications," Applied Mathematics and Computation, vol. 187, no. 1, pp. 250-265, 2007. 

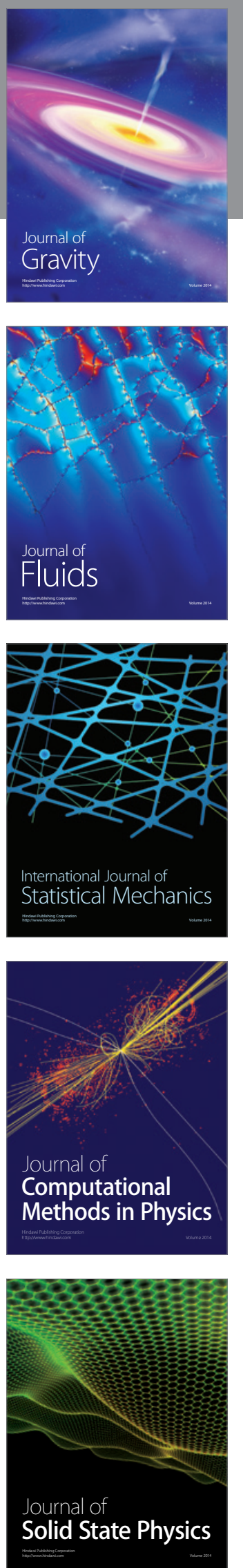

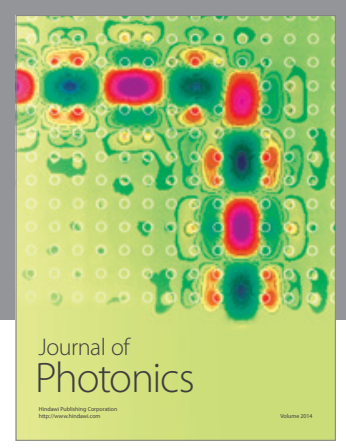

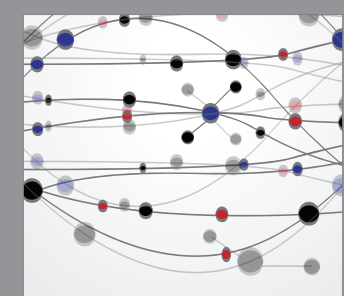

The Scientific World Journal
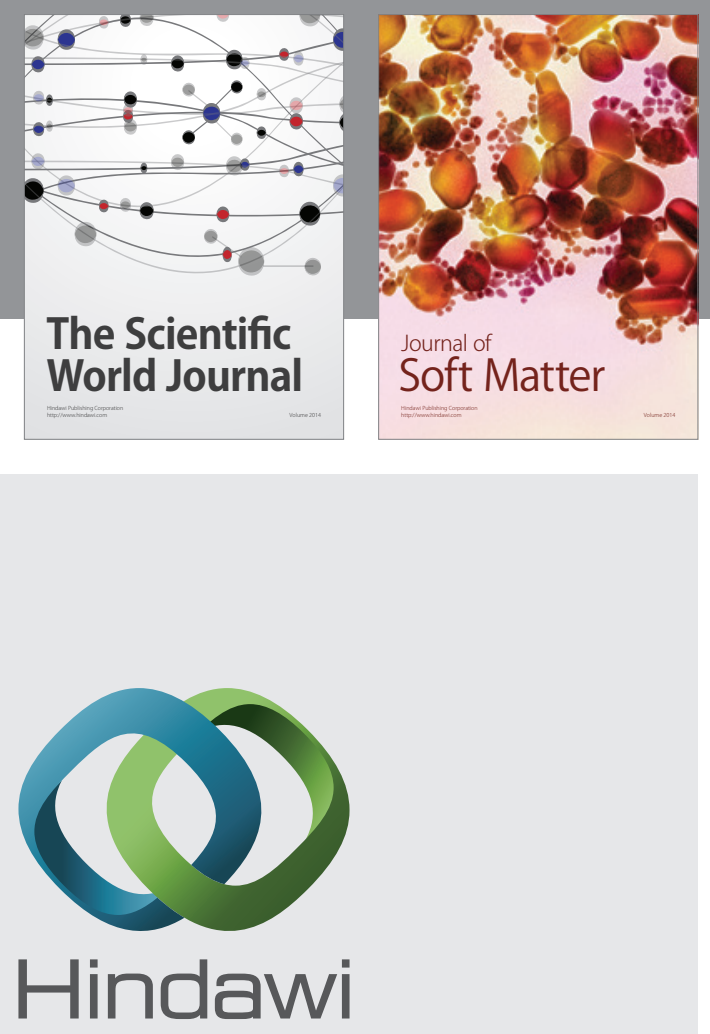

Submit your manuscripts at

http://www.hindawi.com
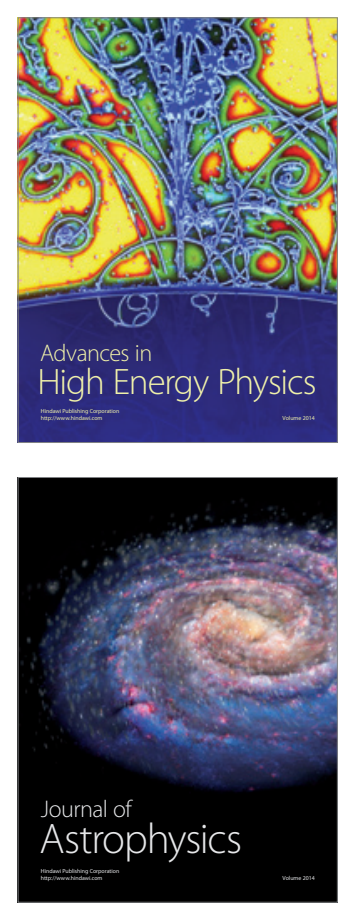
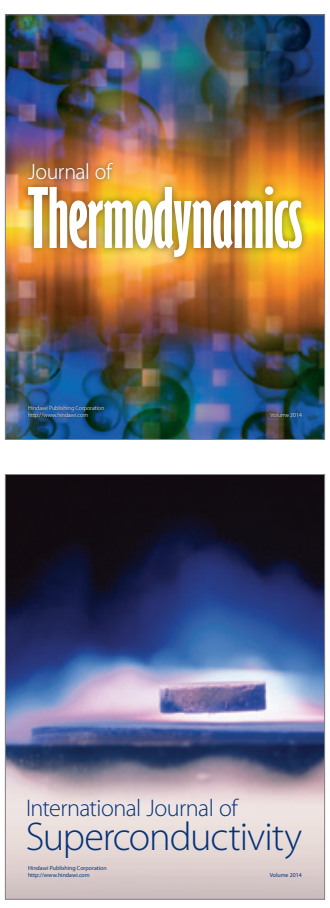
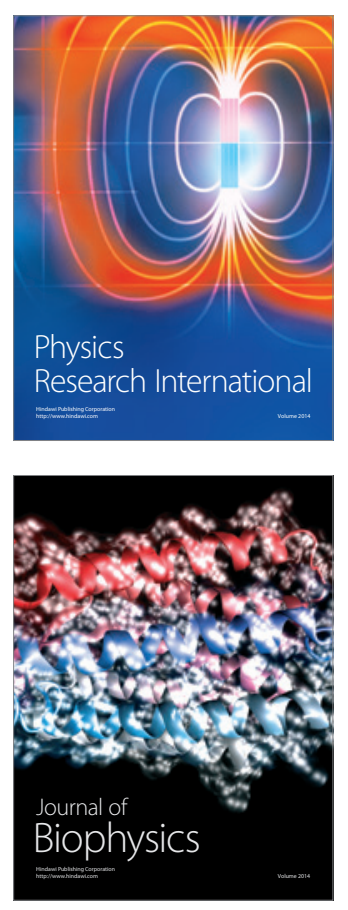
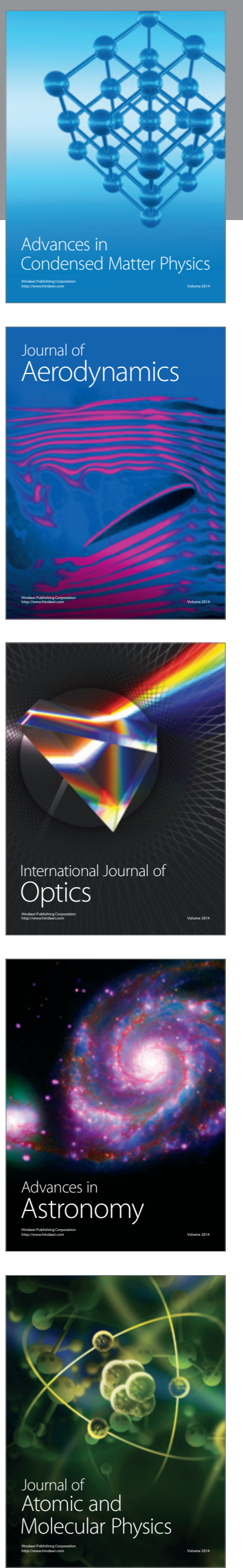